\title{
Plume-Induced Effects on the Near-Wake Region of a Generic Space Launcher Geometry
}

\author{
D. Saile* and A. Gülhan ${ }^{\dagger}$ \\ DLR - German Aerospace Center, Cologne, North Rhine-Westphalia, 51147, Germany
}

\begin{abstract}
The near-wake flow of a generic space launcher geometry with jet is investigated experimentally in the hypersonic flow regime at Mach 6 and an unit Reynolds number of $16 \cdot 10^{6}$ by means of unsteady pressure measurements. This corresponds to an altitude of $50 \mathrm{~km}$ for an Ariane V-like launcher with an underexpanded nozzle flow. The investigations with a truncated ideal contoured wind tunnel model nozzle designed for Mach number of 2.65 is used to simulate the nozzle flow. The objective of this study is the characterization of the plume-induced loads on the main structural components in the near wake region during flight. A parametric study with the reservoir pressures of $11,14,17$ and 20 bar is presented. The transducers capture the pressure signal in the radial and azimuthal direction on the base and on the surface of the nozzle. The signals are spectrally analyzed with the objective to characterize and identify unsteady flow effects in order to reduce base drag and vibrations of future space launchers. The base-pressure spectra exhibits global modes at Strouhal numbers of 0.05 to $0.06,0.09$ to 0.1 and 0.2 to 0.25 , which are attributed in literature to flapping and shedding. Local modes seem to occur

Locally restricted modes can be detected for the tonal disturbances in the high frequency range for transducers on the base closer to the nozzle. The increase of the reservoir pressure shows a consistent increase of the pressure fluctuation level in the base region.
\end{abstract}

\section{Introduction}

Along the ascent trajectory of space launchers, the structural components in the base are exposed to high thermal and mechanical loads. The loss of the Ariane V ECA at its first flight can be given as an example. The inquiry board published as one of the most probable factors the "non-exhaustive definition of the loads to which the Vulcain 2 engine is subjected during flight". Thus, the qualitative and quantitative characterization of the wake flow is crucial for the development of future space launchers.

Axisymmetric base flows have been widely investigated for over 50 years. First experimental results for compressible subsonic flows on bodies of revolution are reported in Ref. ${ }^{1,2,3}$ Ref. $^{4}$ underlines the difference of a freely reattaching flow in comparison with the reattaching flow on a solid wall in the supersonic flows regime.

In the recent past, a blunt base axisymmetic model in the supersonic flow regime was examined by using Rayleigh/Mie scattering (Ref. ${ }^{5,6,7}$ ) and time-series base-pressure measurements (Ref. ${ }^{8,9}$ ). The instantaneous planar Mie scattering images reveals large-scale structures in the shear layer. The analysis of the pressure fluctuations shows a distinct peak at the non-dimensional frequency of $S r_{D}=0.05$ for the transducer located in the center of the base and $S r_{D}=0.1$ for the outermost locations.

Experiments with an Ariane 5-like generic wind tunnel and a nozzle extension are reported in Ref. ${ }^{10}$ for the high subsonic flow regime. A Strouhal number of $S r_{D}=0.08$ are shown for the blunt body, $S r_{D}=0.18$ for a short nozzle within the recirculation region and a broad-band signal centered at $S r_{D}=0.1$ for a nozzle exhibiting the flow reattachement on the nozzle. A realistic and detailed model of the Ariane 5 with two boosters is examined in Ref. ${ }^{11}$ in the high subsonic flow regime. A distinct peak and a broad-band oscillation

\footnotetext{
* Scientific employee, DLR - German Aerospace Center, Institute of Aerodynamics and Flow Technology, Supersonic and Hypersonic Technology Department (AS-HY, Linder Höhe, 51147 Cologne/Germany.

${ }^{\dagger}$ Head of AS-HY, DLR - German Aerospace Center, Institute of Aerodynamics and Flow Technology, Supersonic and Hypersonic Technology Department (AS-HY), Linder Höhe, 51147 Cologne/Germany.
} 
is allocated to $S r_{D}=0.22$ and to a Strouhal number of about 0.5 to 0.6 . In comparable experiments (Ref. ${ }^{12}$ ), fluctuations at $S r_{D}=0.4$ are presented.

In numerical investigations on the blunt-based axisymmetric model for a supersonic flow published in Ref. ${ }^{13}$ and Ref., ${ }^{14}$ large coherent structures are attributed to the unstable modes. Complementary numerical studies (Ref. ${ }^{15}$ ) focused on the shear layer flow. The authors of Ref. ${ }^{16}$ and Ref. ${ }^{17}$ executed numerical studies in the high subsonic region on base flows elongated with an attached smaller cylinder. Characteristic dimensionless frequencies can be found depending on the spatial location within the recirculation bubble.

A common denominator for most studies are fluctuations at about $S r_{D}=0.2$, which is associated with an absolute helicoidal unstable mode. It is considered to be an antisymmetric 'shedding' motion influencing the recirculation region. A coexistance of this convectively unstable mode with the 'flapping' mode is stated in Ref. ${ }^{17}$ Flapping is described in two dimensional flows as an overall growth and decay mechanism of the recirculation region taking place with a Strouhal number of about 0.08 . Fluctuations associated with $S r_{D}=0.6$ are in literature often related to collapsing vortical structures in the shear layer.

Fig. 1 shows the mean flow topology with a focus on the base of an axisymmetric rocket geometry with jet. Upstream from the base, the ambient supersonic flow with the developed boundary layer on the surface encounter an abrupt geometry change due to the edge, which results in an expansion and corresponding deflection of the flow towards the axis. This edge is the starting point of an energetic shear layer. Farther downstream at the nozzle exit plane, the nozzle exit pressure has not fully adapted to the ambient pressure, which causes a further expansion of the jet. The separation from the corner of the nozzle generates a second shear layer. These two shear layers enclose the highly dynamic and subsonic vortex/recirculation region. An external and internal shock are generated at the location where the ambient flow and the nozzle flow interact. This can be seen in (Fig. 3), which is a mean image of a high-speed schlieren measurement derived from experiments of the paper at hand. The cause of the external shock is an adverse pressure gradient in the vortex region due to the displacement effect of the jet. This is where pressure waves emanate and focus to a recompression/external shock.

\section{Methods}

Measurements are conducted in the hypersonic wind tunnel H2K in Cologne at the Institute of Aerodynamics and Flow Technology in the Supersonic and Hypersonic Technology Department. The H2K facility is a blow-down type wind tunnel with a free test section. In order to offer a wide Mach number range up to 11.2 , it is equipped with a vacuum sphere. Depending on which of the six contoured, axissymmetric aerodynamic nozzle is used, the H2K facility can be operated with Mach 4.2, 5.3, 6.0, 7.0, 8.7 or 11.2. The exit diameter of the Mach 6.0 nozzle for the investigations at hand is $600 \mathrm{~mm}$. Eight electric heaters with a maximum electrical power of $5 \mathrm{MW}$ can heat up the air to a reservoir temperature of $1100 \mathrm{~K}$, which is necessary to omit condensation at high Mach numbers and can also be used to adjust the Reynolds number. A maximum unit Reynolds number of about $20 \cdot 10^{6} \mathrm{~m}^{-1}$ is reached at Mach 6 at a mass flow rate of almost $20 \mathrm{kgs}^{-1}$.

The wind tunnel model shown in Fig. 2 is a generic representation of the Ariane 5 with respect to its main geometric features. The wind tunnel model exists as blunt body configuration (configuration B.A) and as configuration with a nozzle (configuration B.C). For the first, investigations have been carried out before (see Ref. ${ }^{18}$ ). This blunt body configuration B.A is used to draw comparisons with the current configuration B.C for the investigations with the interacting nozzle flow. Geometric similarity to Ariane 5 is given with respect to the ratio between the nozzle length and the main body diameter of about 1.2 and a diameter ratio between the main body and the nozzle of about 0.4 .

Simple geometric bodies are used to receive a deeper understanding of the main flow features. The spherical nose features a radius of $10 \mathrm{~mm}$, which is connected to a cone with an apex angle of $36^{\circ}$. The diameter and length of the cylindrical main body is $108 \mathrm{~mm}$ and $328.6 \mathrm{~mm}$, respectively. The wind tunnel model is supported by a strut. The apex angle of the front wedge is $30^{\circ}$, the shaft is $22 \mathrm{~mm}$ wide and $156 \mathrm{~mm}$ long, and the rear wedge has an opening angle of $20^{\circ}$.

The wind tunnel model design is modular with respect to the exterior design - which was described before - and the interior design. The interior design is thermally isolated in order to reach almost thermal equilibrium of all relevant components in the time frame of one run. It consists of a two feeding pipes, a settling chamber with flow straighteners and a TIC-nozzle (truncated ideal contoured) designed for Mach 2.65. The straightener could only be applied up to a reservoir pressure of 14 bar due limitations of its 


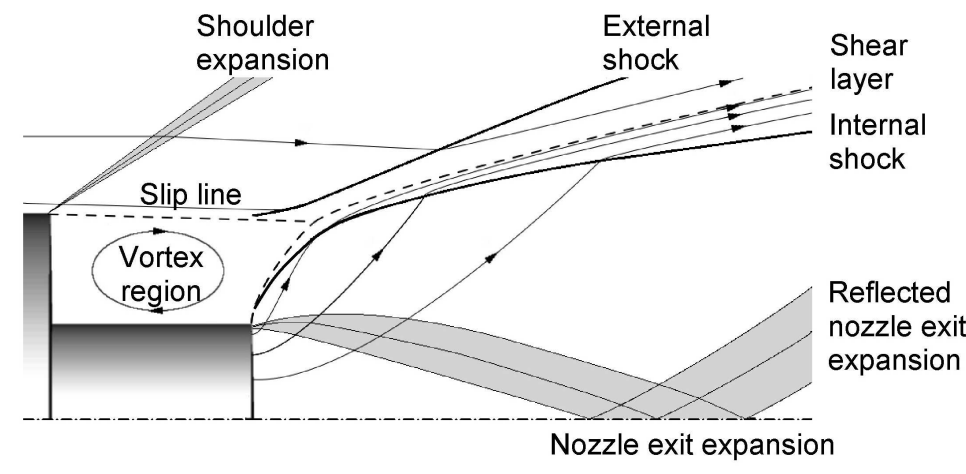

Figure 1. Mean flow topology resulting from the interaction of the base flow with the nozzle flow.

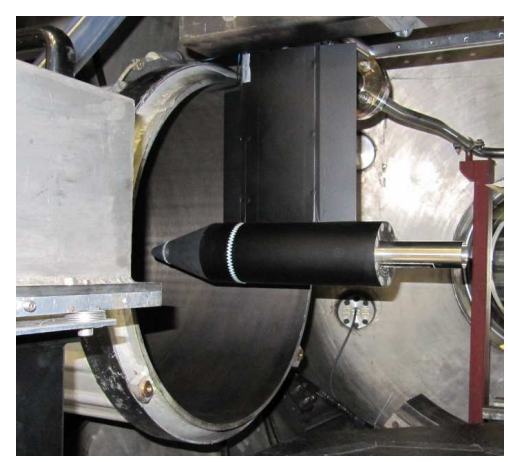

Figure 2. Wind tunnel model in H2K.

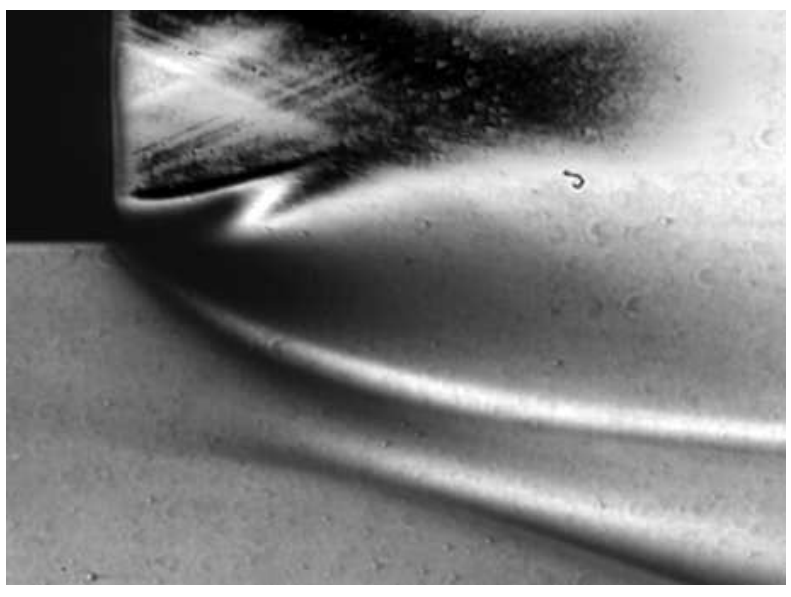

Figure 3. Mean of a high-speed schlieren image.

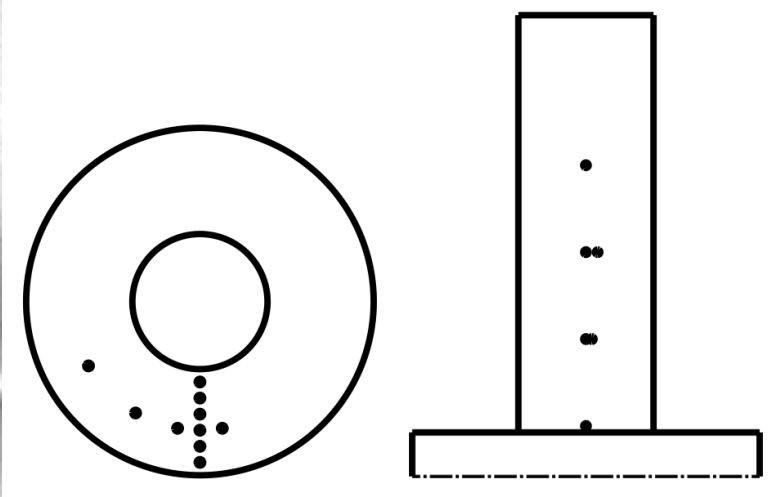

Figure 4. Sketch of transducer positions. 
mechanical strenght. In order to reach similarity to the Vulcain 2 rocket engine of Ariane V, the nozzle is truncated to reach a divergence angle of $\Theta \approx 5^{\circ}$. This results in an average Mach number over the exit area of $M a \approx 2.5$. Details to the nozzle design are given in Ref. ${ }^{19}$ Reservoir pressure and temperature are measured inside the settling chamber.

For the experiments at hand, the H2K facility is operated at Mach 6.0 with a reservoir pressure and temperature of 18 bar and $470 \mathrm{~K}$, which results in an unit Reynolds number of $16 \cdot 10^{6} \mathrm{~m}^{-1}$. The reservoir pressure is varied between to $11,14,17,20$ bar, while the reservoir temperature is kept close to ambient temperature.

The following cylindrical coordinate system is used to describe the positions of the transducers, which are flush mounted on the model surface. The origin of the coordinate system is located in the center of the base. The axis of abscissa $x$ is in the axis of symmetry and points in the downstream direction, and the ordinate describing the radial distance $r$ is set perpendicular pointing in the direction of the strut support. The transducers are positioned on the base in azimuthal and radial direction and on the nozzle on the strutaverted side in the streamwise direction. In total, 16 transducers are used of four different types. An overview is given in Tab. 1. Cylindrical transducers are denoted with XCQ-080, the surface mounted with $L E-062$. The denotation behind describes the pressure range ( 0.35 and $0.7 \mathrm{bar})$ and if the pressure is measured as an absolute value or differentially. The pressure signal is recorded with a sampling rate of $100 \mathrm{kHz}$ and a resolution 24 bit by using a NI PXIe-4331 bridge module. In total, $2 \cdot 10^{6}$ samples are captured per run.

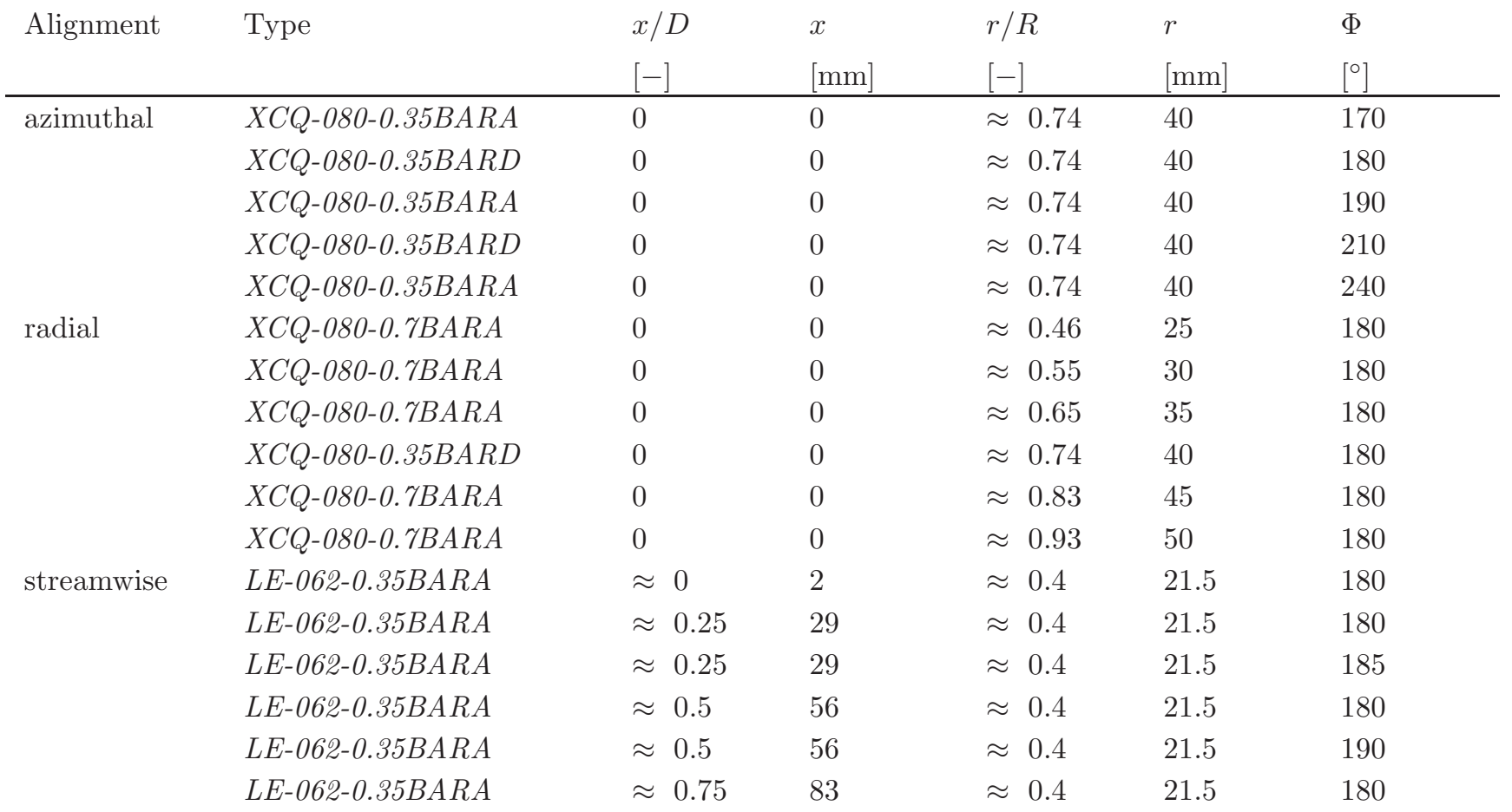

Table 1. Overview to transducer types and positions.

The pressure signal is analyzed with respect to its spectral information and the coherence of the signals. The power spectral density is computed for blocks of 8192 samples, which are filtered with a rectangular window and the evaluation of the whole sequence is executed with an overlap of $50 \%$. Further, the coherence of the various transducers to the transducer at $(r / R, \Phi)=\left(0.46,180^{\circ}\right)$ is found by using Welch's averaged, modified periodogram method. This transducer is selected since it is located in the corner, which is in the vicinity of the transducers on the base and on the nozzle. Additionally, this transducer records most of the dominant modes found from the spectral analysis. Thus, it is suitable for a comparision with respect to the coherence of these modes. As before, the sequence is divided into blocks of 8192 samples on which a hanning window is applied. The blocks in the sequence are shifted by $50 \%$ for each analysis. The frequency is converted into the non-dimensional Strouhal number according to $S r_{D}=f D U_{\infty}^{-1}$, where $f, D$ and $U_{\infty}$ refers to the frequency, the base diameter and the ambient flow velocity, respectively. 


\section{Results}

The spectral density of the base pressure is analyzed with respect to two different foci. First, the spatial distribution in the radial and azimuthal direction on the base and along the nozzle is discussed for a wind tunnel model stagnation pressure of 20 bar. Then, the influence of the wind tunnel model reservoir pressure is shown for three selected transducers. The frequency information above $S r=1.1$ of the spectra is not discussed since it is considered as not relevant from a technical point of view. Aspects of three-dimensionality and of the incoming boundary layer have been discussed in previous studies by Ref. ${ }^{18}$

As it can be seen later on, certain dominant frequency bins seem to be related since they appear and grow as a group. Consequently, the related bins are bundled for the discussion. This categorization has to be taken with care. It is intended as an orientation to simplify the discussion about the various frequency bins. The groups are referred to as $g_{S r_{D}}$, where the subscript $S r_{D}$ is replaced with the frequency range of the peaks. The grouping is shown in Tab. 2.

$$
\begin{aligned}
& g_{0.39-0.48}=[0.39,0.406,0.454,0.48] \\
& g_{0.55-0.59}=[0.563,0.574,0.584] \\
& g_{0.61-0.68}=[0.61,0.63,0.645,0.674] \\
& g_{0.73-0.92}=[0.735,0.84,0.8755,0.905,0.914] \\
& g_{0.93-1.01}=[0.93-0.94,0.96,0.987,1.01]
\end{aligned}
$$

Table 2. Grouping of frequency peaks.

Fig. 5 shows pressure spectra on the base at various azimuthal locations $\left(\Phi=170^{\circ}, 180^{\circ}, 190^{\circ}, 210^{\circ}\right.$, $240^{\circ}$ ) at $r / R=0.74$. In the low frequency range, the overall trend of all curves with a broadband signal at about $0.05-0.06,0.9$ to $1.0,0.2$ to 0.25 is comparable. The broadband signal is weakly developed, thus, the allocation of the peak to a certain frequency is over a wider range. The spectra of the neighboring transducers at $\Phi=170^{\circ}, 180^{\circ}$ and $190^{\circ}$ actually coincide for the observed frequency range. With angles $\Phi$ closer to the strut, a decrease of the power spectral density can be observed. In the higher frequency range, the spectra feature tonal peaks of group $g_{0.73-0.92}$ and $g_{0.93-1.01}$, which can be detected at all azimuthal positions. The transducers closer to the strut exhibit some additional peaks in the range of $g_{0.61-0.68}$.

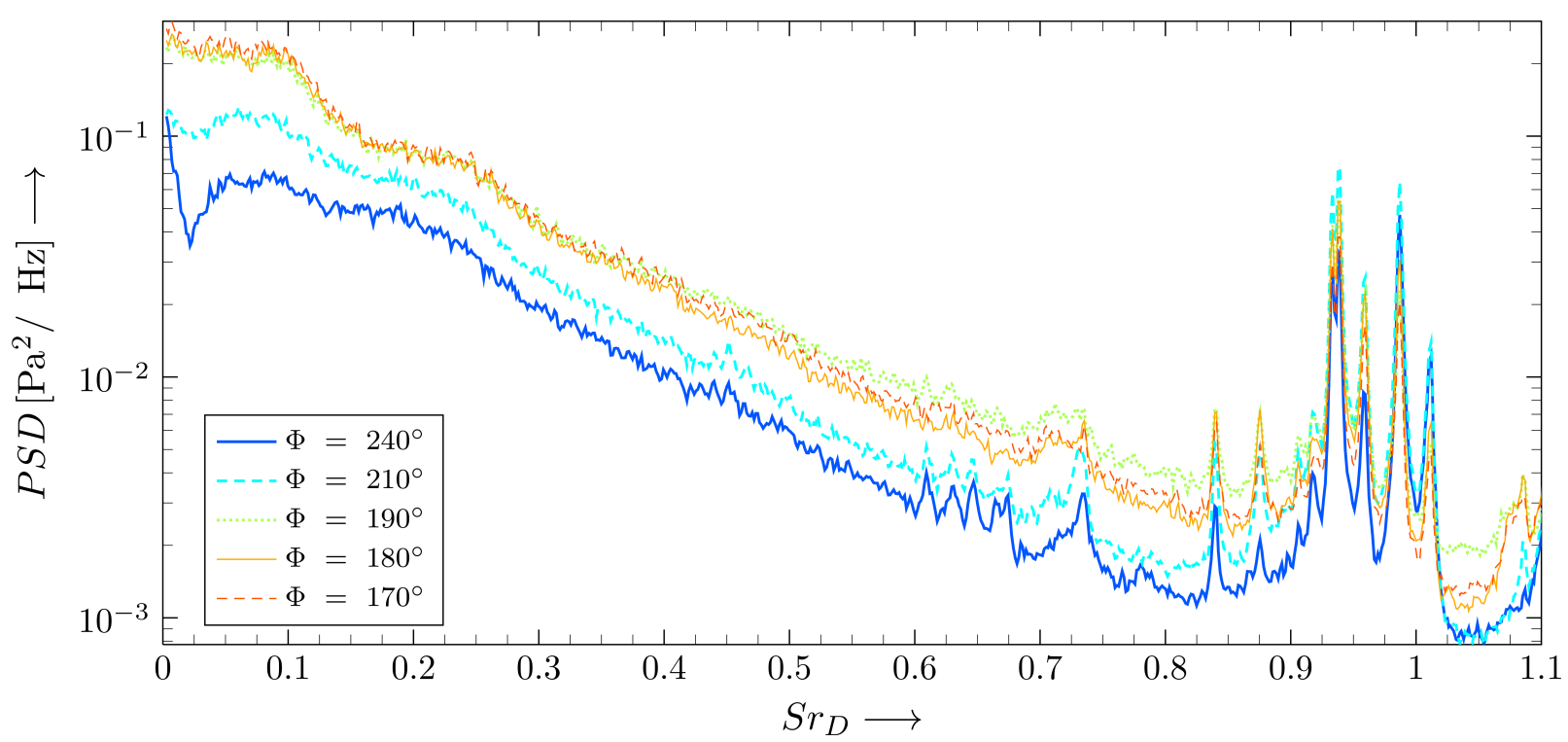

Figure 5. Power spectral density on the base for different azimuthal positions $\Phi$ (at position $r / R=0.74$ ) for a wind tunnel stagnation pressure of $p_{0}=20$ bar.

The power spectral density of the transducers in the radial direction (Fig. 6) exhibit a course, which 
is clearly comparable to the azimuthal alignment in the low frequency range. In fact, the curves at the outskirts of the base coincide again. Also, it can be seen that the transducer closest to the edge of the base $(r / R=0.93)$ exhibits weaker oscillations in the high frequency range, which are assigned to the group $g_{0.93-1.01}$. By approaching to the corner, one can detect an overall increase of the power spectral density, and more dominant frequencies are successively added to the spectra. First, the group $g_{0.73-0.92}$ is added $(r / R=0.74)$, then the group at $g_{0.39-0.48}$ and $g_{0.61-0.68}$.

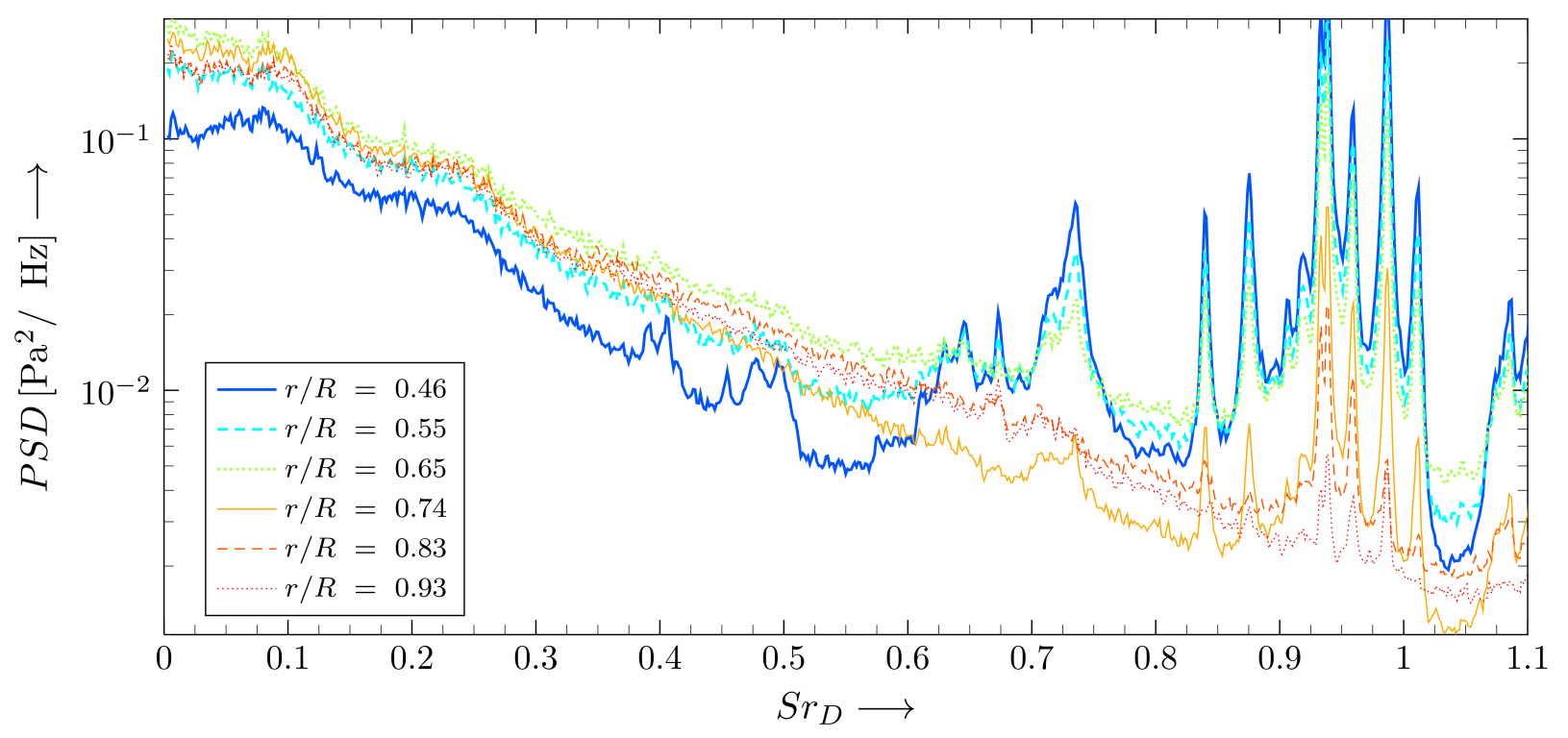

Figure 6. Power spectral density on the base for different radial positions $r$ (at position $\Phi=180)$ for a wind tunnel stagnation pressure of $p_{0}=20 \mathrm{bar}$.

The spectra of the transducers on the nozzle are shown in Fig. 7. It can be seen that the spectrum of the transducer on the nozzle in the corner between base and nozzle shows strong similarities to the transducer closest to the corner on the base as seen in Fig. $6(r / R=0.46)$. Both transducers sense the broadband signal at 0.05 to 0.06 and 0.2 to 0.25 and all the tonal signals mentioned before in the high frequency range. Though, the group at $g_{0.39-0.48}$ is remarkably stronger. With increasing distance from the base, the spectra become flatter, but higher concerning to the level. At $x / D \approx 0.5$, only tonal oscillations of the group $g_{0.73-0.92}$ are notable. At that position, even the broadband signal at 0.05 to 0.06 disappears, while a broadband signal at about 0.6 and 0.87 seems to appear. For the transducer closest to the edge of the nozzle $(x / D=0.75)$, the tonal signals mostly disappeared, while a broadband oscillation can be found at about 0.93

The power spectral density plots indicate coherent signals, thus, the following plots are intended reveal some of these correlations. As mentioned before, the coherence is computed with respect to the transducer in the corner of the base $\left(r / R=0.46, \Phi=180^{\circ}\right)$ since it is in the vicinity of the other transducers and shows most of the dominant modes. Fig. 8 shows a predominant coherence to the broadband signal at 0.9 to $0.1,0.2$ to 0.25 and a very high coherence to $g_{0.73-0.92}$ and $g_{0.93-1.01}$. The coherence in the radial direction (Fig. 9) shows similar results. The broadband signal at 0.05 to 0.09 and for 0.2 to 0.25 can be found with a medium coherence for all transducers. The signals in the high frequency range cohere strongly up to a radius of about $r / R=0.65$ and disappears closer to the edge. Fig. 10 reveals a wide dependency up to about 0.4 for the neighboring transducer. Further downstream, only the high frequency information of the group $g_{0.93-1.01}$ is apparent, whereas it also disappears for the transducer closest to the nozzle exit.

In the following, the dependency of the base pressure fluctuation is investigated with respect to reservoir pressure for the nozzle flow. Fig. 11 to 13 depict the power spectral density on the base, in the corner on the nozzle and at a position close to the nozzle exit of the nozzle. In detail, the transducers are located at $(r / R, \Phi)=\left(0.74,180^{\circ}\right),(x, \Phi) \approx\left(0 \mathrm{~mm}, 180^{\circ}\right)$ and $(x / D, \Phi) \approx\left(0.75,180^{\circ}\right)$, respectively. The various plots show runs executed with a reservoir pressure in the model of $p_{0}=0,11,14,17$ and 20 bar. 


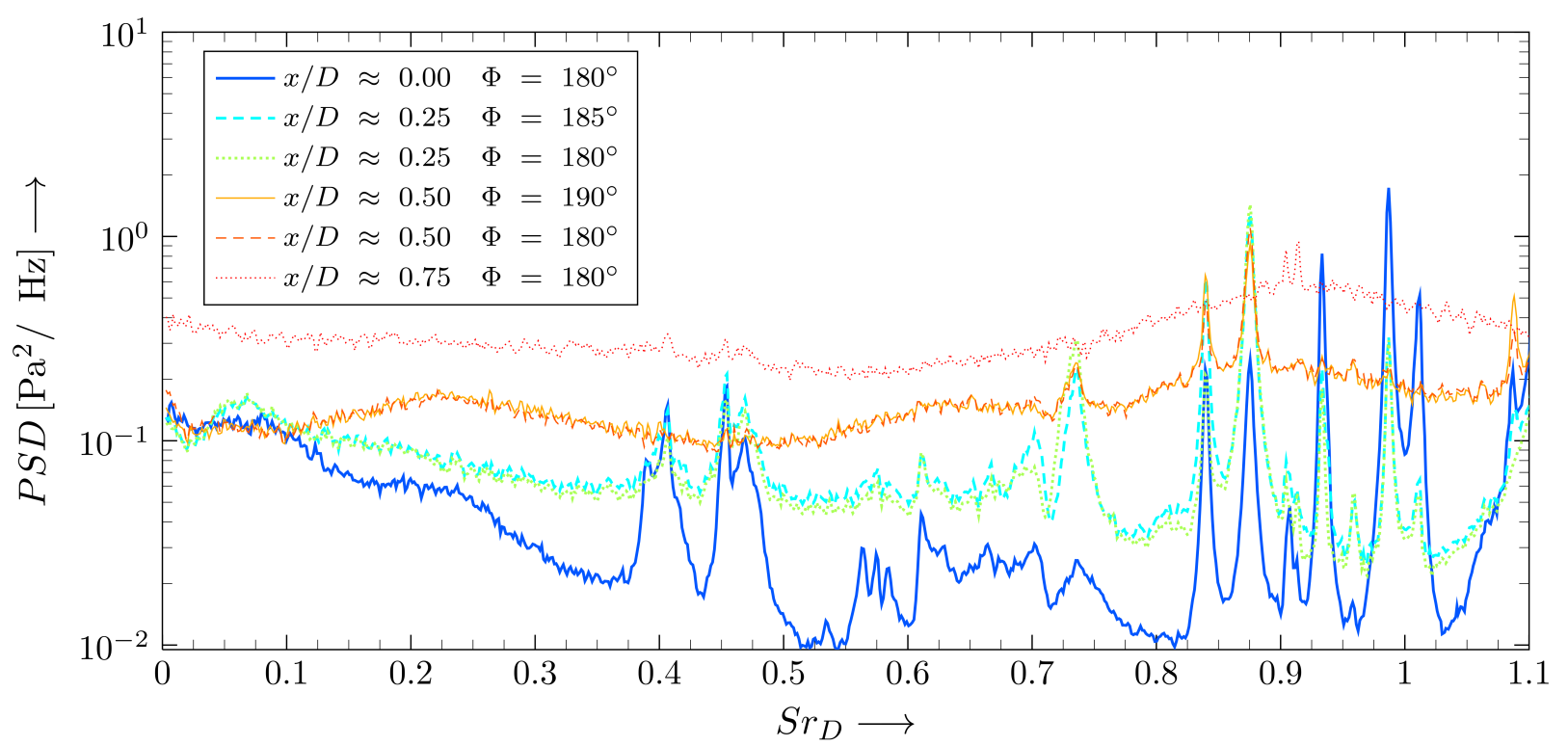

Figure 7. Power spectral density on the nozzle for different positions $x$ downstream of the base for a wind tunnel stagnation pressure of $p_{0}=20 \mathrm{bar}$.

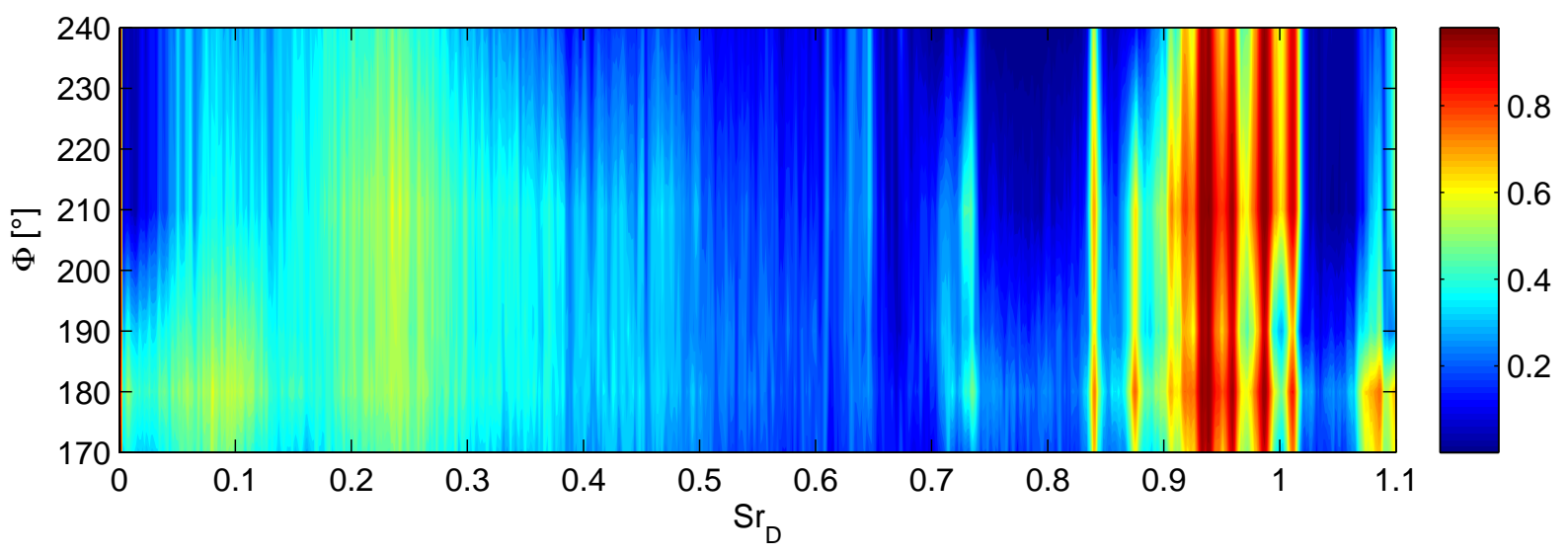

Figure 8. Coherence of the transducers with the azimuthal alignment with respect to the transducer at $r / R=0.46$ and $180^{\circ}$. 


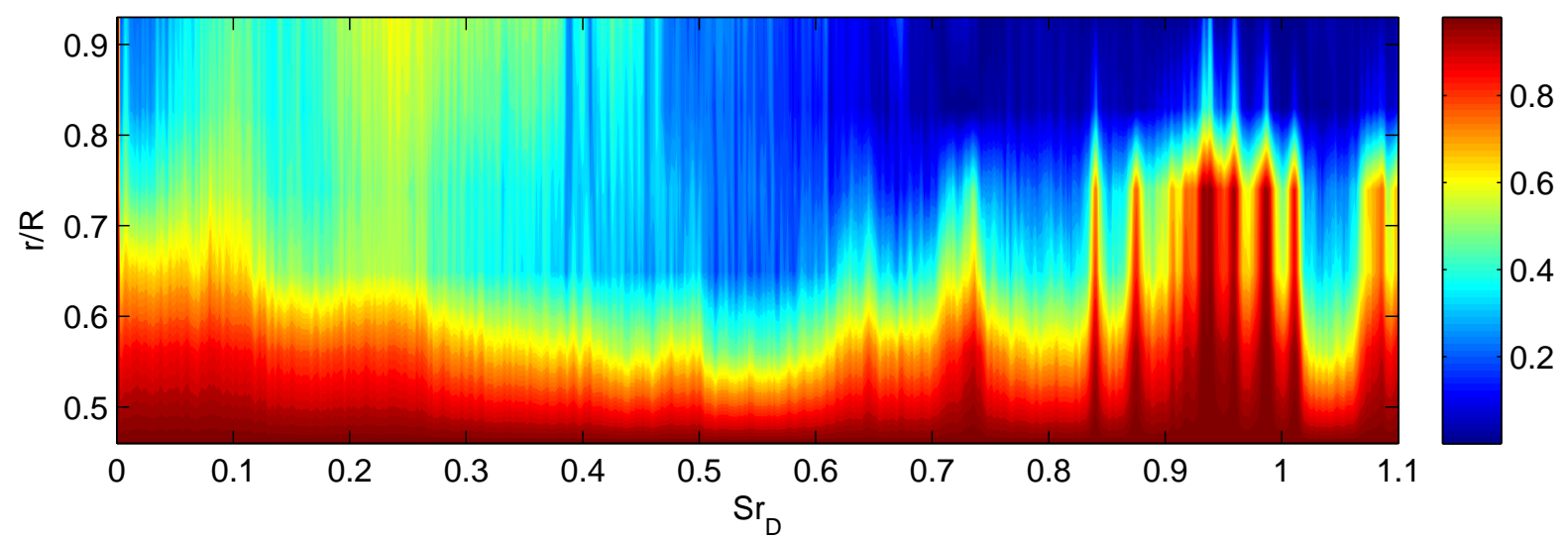

Figure 9. Coherence of the transducers with the radial alignment with respect to the transducer at $r / R=0.46$ and $180^{\circ}$.

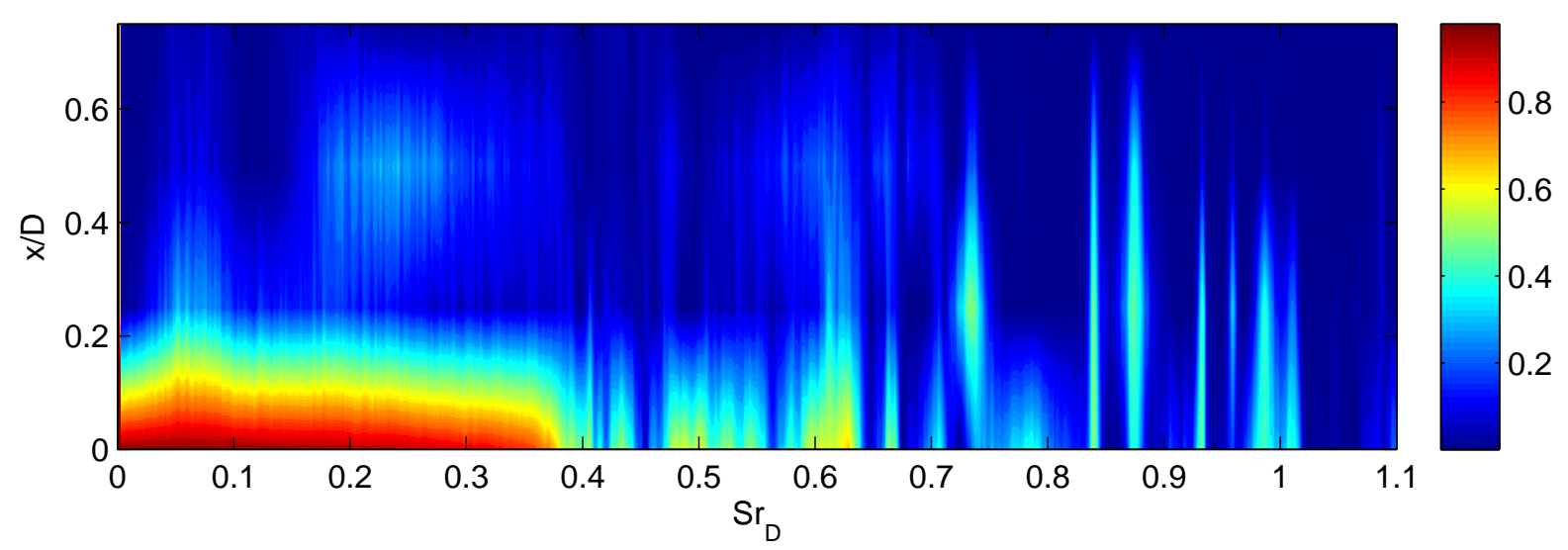

Figure 10. Coherence of the transducers alignment in the stream wise direction with respect to the transducer at $r / R=0.46$ and $180^{\circ}$. 
For the transducer on the base with no plume flow (Fig. 11), the spectrum reveals a broadband signal centering at $S r_{D}=0.27$, which has been observed in previous studies under the same flow conditions (Ref. $\left.{ }^{18}\right)$. No distinct frequency information can be found for in the high frequency range. In comparison, the spectra for the experiments with a plume differ clearly, while a similarity between the different runs with plume is especially detectable in the low frequency range up to 0.6. In this range, the level of the pressure fluctuation is consistently shifted to higher amplitudes with increasing reservoir pressure. The broadband oscillations at about 0.09 to 0.1 and between 0.2 to 0.25 are maintained at the same location. In the higher frequency range, the oscillations grouped in $g_{0.73-0.92}$ and $g_{0.93-1.01}$ appear for all transducers. The runs for a reservoir pressure of 11 and 14 bar feature an additional peak at about 0.665 .

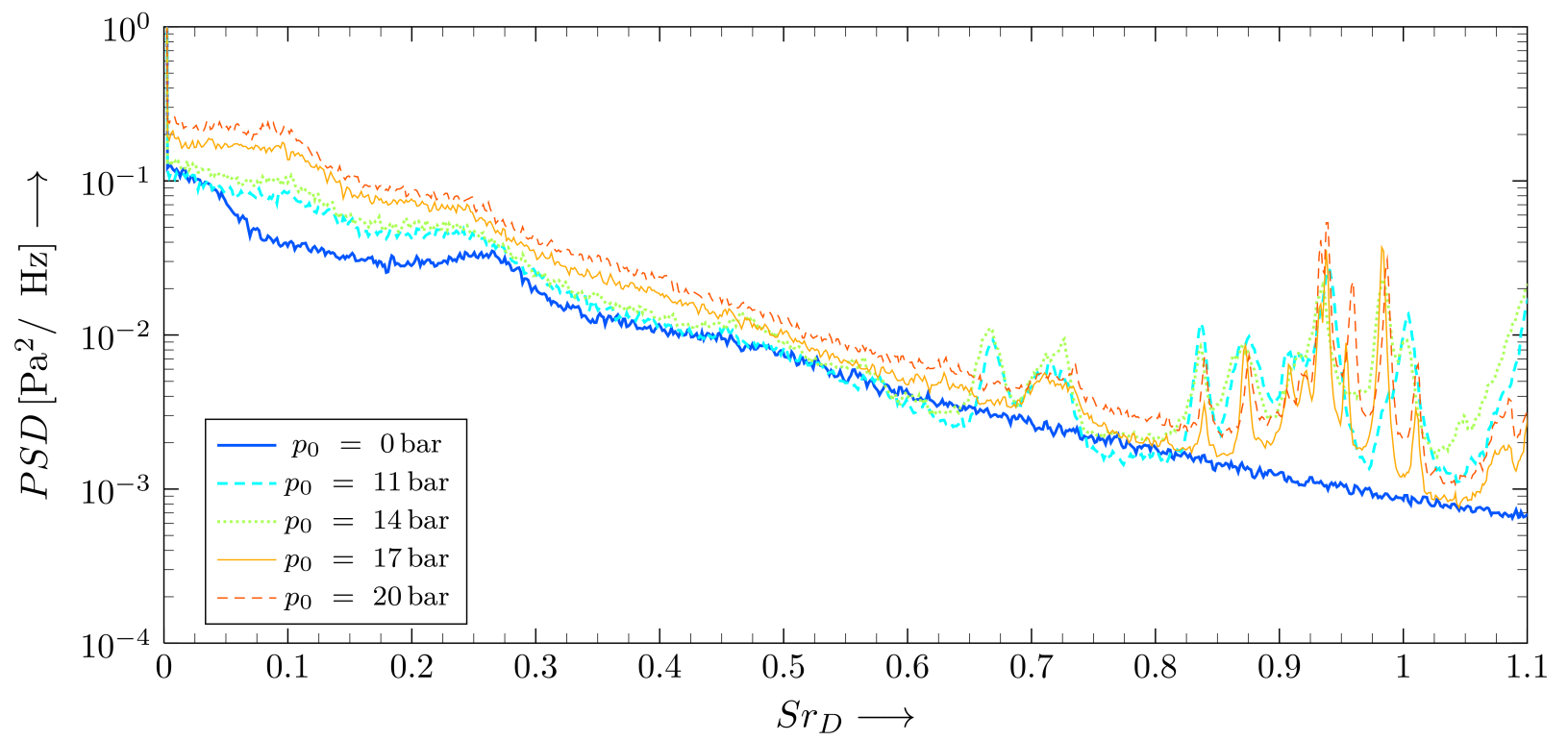

Figure 11. Power spectral density of the pressure on the base (at position $\left.(r / R, \Phi)=\left(0.74,180^{\circ}\right)\right)$ as a function of the wind tunnel model stagnation pressure $p_{0}$.

The opposite is the case for the transducer in the corner on the nozzle (Fig. 12). Here, the spectra of $p_{0}=11$ and 14 bar are missing a peak in the group that is assigned to $g_{0.39-0.48}$. Despite that, the overall course is comparable to the observations before. The power spectral density of the fluctuations increase with increasing reservoir pressure, while the periodicity of the oscillations stays constant.

Notable is the spectra of the last transducer on the nozzle (Fig. 13) since the increase of the power spectral density, which was observed for all transducers before for a higher reservoir pressure, is not detectable in the low frequency range. The spectra exhibits the same level up to a Strouhal number of 0.55. In the higher frequency range between 0.7 and 1.1, a broadband signal with a clear dependency to the reservoir pressure is visible. The increase of the reservoir pressure results in a shift of this broadband signal to lower frequencies with an increasing power spectral density level. Again, the spectra show oscillations, which correspond with the removal of the straightener in the settling chamber (at about 0.22 and at about 0.91).

\section{Discussion}

The results presented here show that there exists a spatial distribution of the pressure oscillations for the investigated configuration. Note, that the signals of the transducers seem to be highly reliable since curves of neighboring transducers coincide (e.g Fig. 8, Fig. 10). The fluctuations seem to be driven by modes that affect the recirculation region in a global manner and also by rather locally restricted modes. Broadband oscillations with a periodicity of about $S r=0.05$ to $0.06,0.09$ to 0.1 and 0.2 to 0.25 are measured predominantly by all transducers on the base and in the corner on the nozzle. 


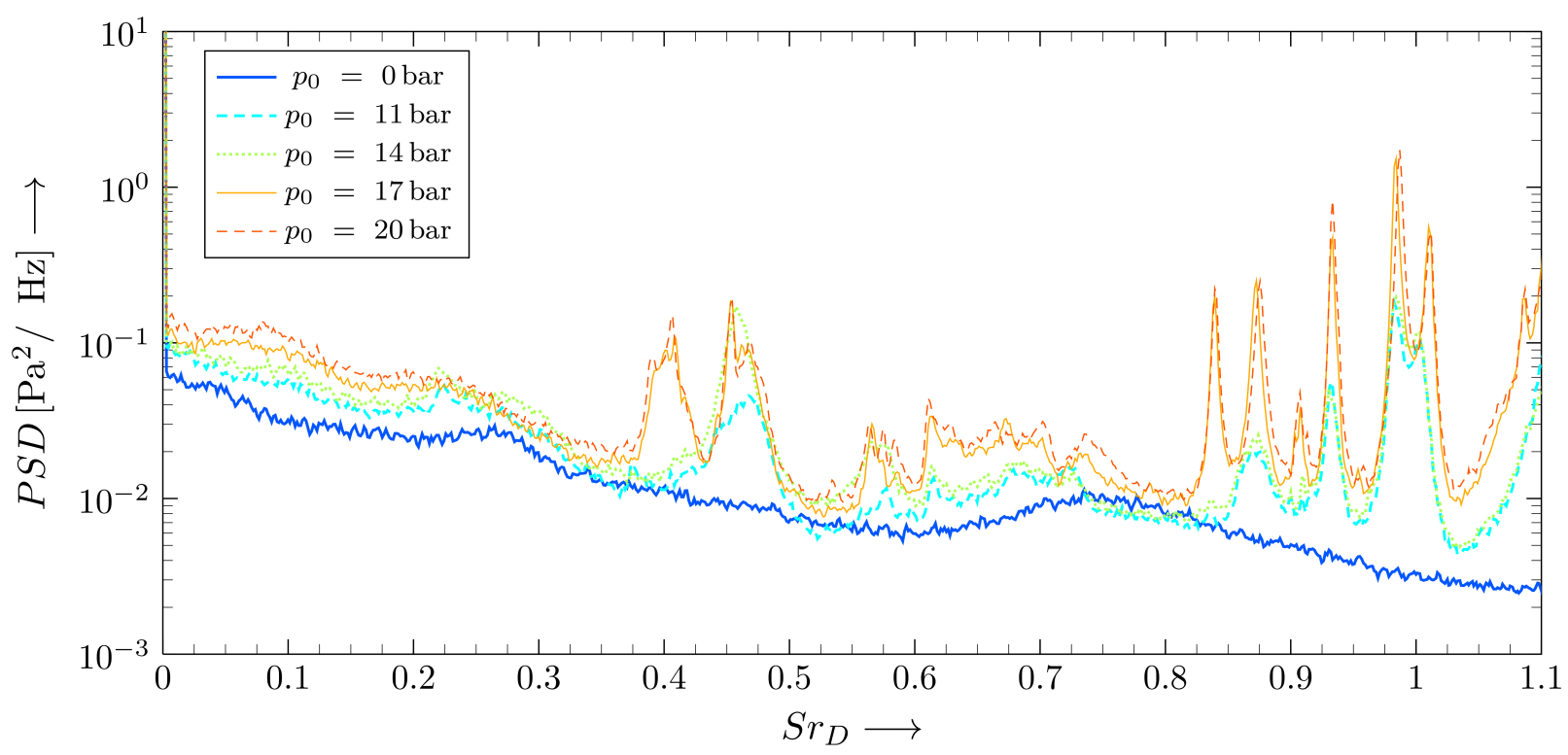

Figure 12. Power spectral density of the pressure in the corner between base and nozzle (at position $\left.(x, \Phi) \approx\left(0 \mathrm{~mm}, 180^{\circ}\right)\right)$ as a function of the wind tunnel model stagnation pressure $p_{0}$.

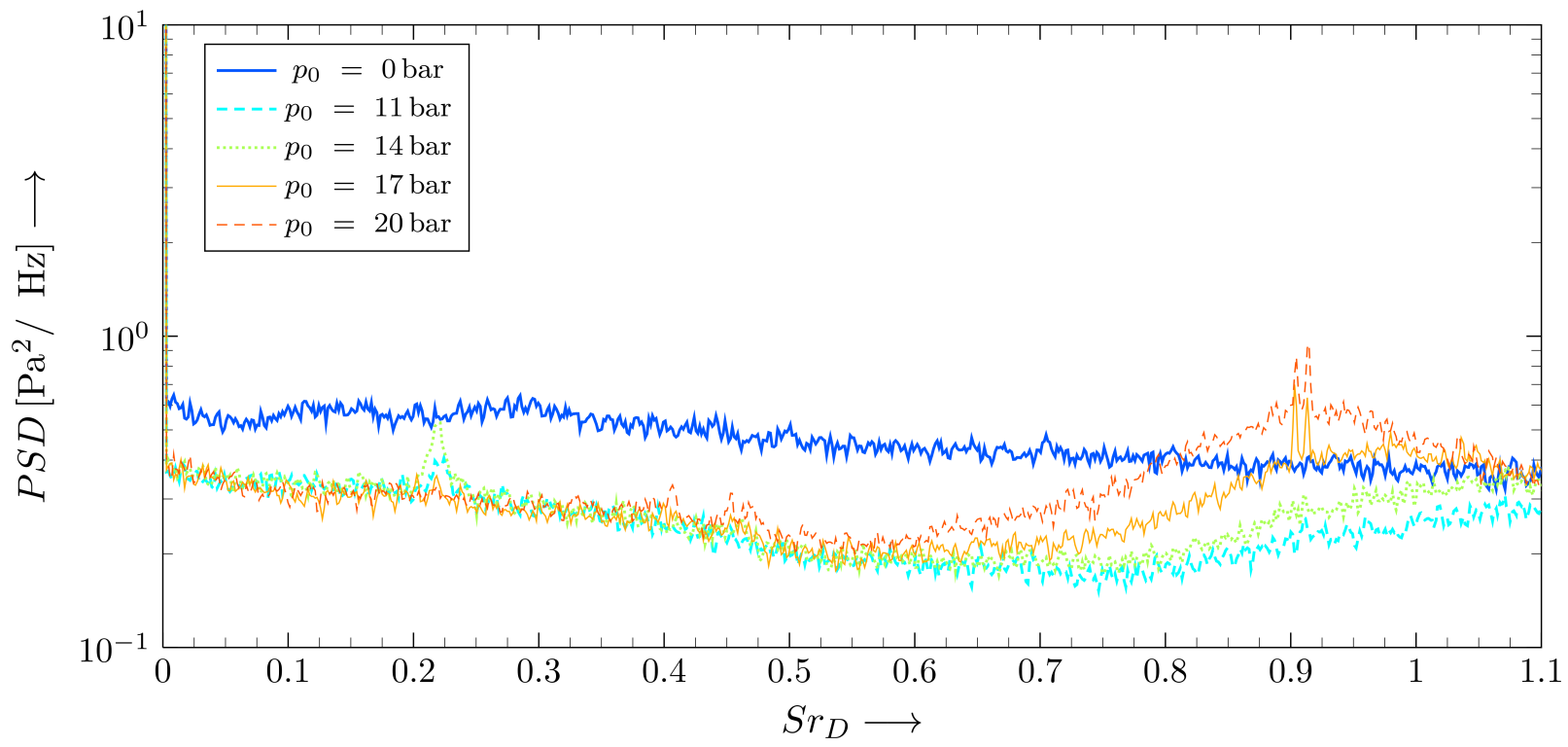

Figure 13. Power spectral density of the pressure on the nozzle (at position $(x / D, \Phi) \approx\left(0.75,180^{\circ}\right)$ ) as a function of the wind tunnel model stagnation pressure $p_{0}$. 
In comparison to literature, the before mentioned Strouhal numbers can be found repeatedly. For instant, in axisymmetric, blunt-based wake flow investigations in the supersonic regime, Strouhal numbers of $S r_{D}=$ 0.05 and $S r_{D}=0.1$ were found for the center and for the outermost locations (Ref. ${ }^{8,9}$ ), respectively. Previous in house studies for the blunt base configuration in the hypersonic regime of Ref. ${ }^{18}$ revealed the same dominant frequencies in the hypersonic flow regime. A Strouhal number of about 0.08 is assigned to the "flapping" instability, which describes the overall growth and decay mechanism of the recirculation region. A Strouhal number of 0.1 can also be found in Ref. ${ }^{10}$ for a flow reattached on a nozzle. Ref. ${ }^{17}$ reports of a coexistence of that instability mode with the well-known shedding instability occurring at a Strouhal number of about $S r_{D}=0.2$. A distinct peak at 0.22 is found in experiments of Ref. ${ }^{11}$ to the pressure fluctuations of Ariane V in the subsonic regime.

Indications for local effects can be found in Fig. 6. The oscillations extend successively over a wider frequency range with a decreasing radial distance. A locally confined, highly dynamic secondary corner vortex could be an explanation for such a behavior. This hypothesis is supported by Fig. 8. It shows on the one hand, that the transducer in the azimuthal direction are equally affected, but on the other hand, that the modes grouped in $g_{0.39-0.48}$ and $g_{0.61-0.68}$ are either completely missing or small. This is in opposition to the transducer in the corner on the nozzle (Fig. 10), that features the discussed frequencies again. It seems like only the corner flow is excited in a certain frequency range, and thus, evidence a secondary corner vortex causing fluctuations associated with the group $g_{0.39-0.48}$ and $g_{0.61-0.68}$.

Generally, the tonal disturbances in the spectra only occur in the presence of the plume (e.g. Fig. 6, Fig. 12). Though, the transducer closest to the nozzle exit shows no such oscillations (Fig. 13) raising the question where these frequencies come from. If they are only induced by the displacement effect of the plume or how they travel upstream from nozzle exit without passing this transducer. No satisfactory answer is found to that question yet. The shear layer is not considered to be a pathway for this information since it is supersonic, and it seems like structure-borne sound can be excluded too since it would be present throughout most transducers on the nozzle or base.

Similar studies (Ref. ${ }^{20}$ ) to the dynamic bevior of the recompression shock with a plume showed and oscillation of external recompression shock with 5000 to $6000 \mathrm{~Hz}$, which corresponds to a Strouhal number of $S r_{D}=0.53$ to 0.64 . Frequencies in the same range can also be found in the study at hand suggesting that the pressure fluctuations are related to effects also seen in the behavior of the recompression shock. Slight discrepancies in the spectra Fig. 11 to Fig. 13 with respect to occurring frequencies hint to a dependency to activities in the plume. The runs with 11 and 14 bar are executed with straighteners, while 17 and 20 bar are not. This might be reflected in the spectra (e.g. in Fig. 11 at $S r_{D}=0.4$ ).

Another local effect refers to azimuthal modes as found in the investigations by Ref. ${ }^{21}$ These numerical simulations show an unsteady static pressure distribution on the base with alternating patches of high and low pressure areas with a wavelength of 5 to 6 around the base. A dependency of the pressure fluctuations to the azimuthal location can also be detected in Fig. 8, where the overall level of the power spectral density decreases with angles closer to the strut. But, for the case here, the assignment to azimuthal modes might be bias since the strut support possibly prevents a free organization of the flow on the base and introduces a preferred orientation of modes on the base.

The parametric study with increasing reservoir pressure shows that the power spectra density are consistently increasing on the base and on the nozzle close to the base (Fig. 11, Fig. 12). The higher reservoir pressure results in a higher impulse of the jet, which is assumed to be responsible for a stronger displacement effect causing presumably a stronger entrainment in the recirculation region. More energy is transported into recirculation region causing the observed higher pressure fluctuations. The pressure fluctuations closer to the exit of the nozzle (Fig. 13) are independent to that pressure increase in the low frequency range. It seems like in that range, the strength of the pressure fluctuations is dominated by the ambient flow, which is kept constant. In the high frequency range, an increasing reservoir pressure results in an increasing power spectral density levels accompanied with a shift to lower frequencies. This might also be caused by the stronger displacement effect, which forces the adverse pressure gradient of the readapting flow to travel further upstream.

\section{Conclusions}

The present work is in line with the previous studies on the near-wake region of generic space launcher geometries Ref. ${ }^{18}$ The base pressure fluctuations of an axisymmetric rocket model with jet is investigated 
at Mach 6 for a Reynolds number of $1.7 \cdot 10^{6}$ with respect to the base diameter. The wind tunnel model is equipped with a TIC-nozzle with a design Mach number of 2.65 and investigated for a reservoir pressure of $11,14,17,20$ bar and no nozzle flow at a reservoir temperature according to the ambient temperature. The pressure in the near-wake region is investigated by analyzing the power spectral density of the signals on the base and on the nozzle. Additionally, the coherence of all pressure signals to a selected transducer on the base is studied.

The results show that the jet has a strong impacts on the base pressure fluctuations. The measurements reveal global modes at 0.05 to $0.06,0.09$ to 0.1 and 0.2 on the base. The last two modes are associated in literature with flapping and shedding, respectively. Additionally and in contrast to the situation with no jet, high frequency information can be found in the spectra for the transducers close to the base. Generally, the fluctuations are dependent on the location. The dynamic range increases for transducers closer to the nozzle, which indicates a secondary corner vortex. In the azimuthal direction, the spectra feature the same trend with essentially the same peaks, but different levels. This either indicates an azimuthal mode or a preferred orientation of the flow due to the strut support. The systematic investigation of a reservoir pressure increase revealed a corresponding amplification of the base pressure fluctuations. The transducer, which is assumed to be close to the recompression region, senses a shift to lower frequencies with stronger fluctuations for higher reservoir pressures. In the future, the results discussed here will be complemented with high-speed schlieren investigations to gain deeper insights into the dynamic behavior and the coupling between base pressure fluctuations and the unsteady behavior of the recompression shock. Additionally, the influence of high temperature jets and also geometry will be investigated.

\section{Acknowledgement}

Financial support by the German Research Foundation (Deutsche Forschungsgemeinschaft - DFG) in the framework of the Sonderforschungsbereich Transregio 40 is gratefully acknowledged. Thanks to the technical stuff of the supersonic and hypersonic department for the excellent support.

\section{References}

${ }^{1}$ Eldred, K., "Base Pressure Fluctuations," Journal of the Acoustical Society of America, , No. 33, 1961, pp. 59-63.

${ }^{2}$ Mabey, D., "Some Measurements of Base Pressure Fluctuations at Subsonic and Supersonic Speeds," Aeronautical Research Council; also Royal Aircraft Establishment, RAE-TR-70148, Bedford, England, U.K., August 1970, 1972.

${ }^{3}$ Shvets, A., "Base Pressure Fluctuations," Fluid Dynamics, Vol. 14, No. 3, 1979, pp. 394-401.

${ }^{4}$ Délery, J. and Sirieix, M., "Base Flows behind Missiles," AGARD LS-98, T.P. no. 1979-14E.

${ }^{5}$ Herrin, J. and Dutton, J., "The Turbulence Structure of a Reattaching Axisymmetric Compressible Free Shear Layer," Physics of Fluids, Vol. 9, No. 11, 1997, pp. 3502-3512.

${ }^{6}$ Bourdon, C. and Dutton, J., "Visualizations and Measurements of Axisymmetric Base Flows Altered by Surface Disturbances," 39 $9^{\text {th }}$ AAIA Aerospace Sciences, Meeting and Exhibit, 08-11 January 2001, AIAA 2001-0286, Reno, Nevada, USA, 2001.

${ }^{7}$ Cannon, P., Gregory, S. E., and Dutton, J., "Time-Series Analysis Axisymmetric Base-Pressure Measurements with Simultaneous Near-Wake Planar Visualizations," 5th AIAA Fluid Dynamics Conference and Exhibit, AIAA Paper 2005-5285, 2005.

${ }^{8}$ Janssen, J. and Dutton, J., "Time-Series Analysis of Supersonic Base-Pressure Fluctuations," AIAA Journal, Vol. 42, No. 3, 2004, pp. 605-613.

${ }^{9}$ Janssen, J. and Dutton, J., "Sub-Boundary-Layer Disturbance Effects on Supersonic Base-Pressure Fluctuations," Journal of Spacecraft and Rockets, Vol. 42, No. 6, 2005, pp. 1017-1024.

${ }^{10}$ Deprés, D., Reijasse, P., and Dussauge, J., "Analysis of Unsteadiness in Afterbody Transonic Flows," AIAA Journal, Vol. 42, No. 12, 2004.

${ }^{11}$ Deprés, D., Radulovic, S., and Lambare, H., "Reduction of Unsteady Effects in Afterbody Transonic Flows," $6^{\text {th }}$ International Symposium on Launcher Technologies, Munich, 2005.

${ }^{12}$ David, C. and Radulovic, S., "Prediction of Buffet Loads on the Ariane 5 Afterbody," $6^{\text {th }}$ International Symposium on Launcher Technologies, Munich, 2005.

${ }^{13}$ Sandberg, R. and Fasel, H., "Direct Numerical Simulations of Transitional Supersonic Base Flows," AIAA Journal, Vol. 44, No. 4, 2006, pp. 848-858.

${ }^{14}$ Sandberg, R. and Fasel, H., "Numerical Investigation of Transitional Supersonic Axisymmetric Wakes," Journal of Fluid Mechanics, Vol. 563, 2006, pp. 1-41.

${ }^{15}$ Simon, F., Deck, S., Guillen, P., Sagaut, P., and Merlen, A., "Numerical Simulation of the Compressible Mixing Layer Past an Axisymmetric Trailing Edge," Journal of Fluid Mechanics, 591, pp. 215-253.

${ }^{16}$ Deck, S. and Thorigny, P., "Unsteadiness of an Axisymmetric Separating-Reattaching Flow: Numerical Investigation," Physics of Fluids, Vol. 6, 19, pp. 1017-1024. 
${ }^{17}$ Weiss, P.-É., Deck, S. R., J.-C., and Sagaut, P., "On the Dynamics of Axisymmetric Turbulent Separating/Reattaching Flows," AIAA Journal, Vol. 42, No. 3, 2009, pp. 605-613.

${ }^{18}$ Saile, D., Gülhan, A., and Henckels, A., "Investigations on the Near-Wake Region of a Generic Space Launcher Geometry," $17^{\text {th }}$ AIAA International Space Planes and Hypersonic Systems and Technologies Conference, AIAA 2011-2352, 2011.

${ }^{19}$ Saile, D., Gülhan, A., and Henckels, A., "Design of the TIC-nozzle and Definition of the Instrumentation." Annual Report SFB TRR40 2010, 2010.

${ }^{20}$ Henckels, A. and Gülhan, A., "Experimental Study of the Base Flow," In: Gülhan, A. (Ed.), RESPACE - Key Technologies for Reusable Space Systems, Springer-Verlag Berlin Heidelberg, ISBN 978-3-540-77818-9, 2008, pp. 21-39.

${ }^{21}$ Meiss, J.-H. and Schröder, W., "Large-Eddy Simulation of a Generic Space Vehicle," In: Gülhan, A. (Ed.), RESPACE Key Technologies for Reusable Space Systems, Springer-Verlag Berlin Heidelberg, ISBN 978-3-540-77818-9, 2008, pp. 40-56. 